\title{
Atuação do enfermeiro na prevenção da gangrena de Fournier: atenção à saúde do homem
}

\author{
Carenine Maria Gomes Mota*, Jaciara de Araújo Borges*, Anna Karolina Lages de Araújo**, \\ Nelson Jorge Carvalho Batista, M.Sc. ${ }^{* * *}$
}

${ }^{*}$ Graduandas do Curso de Enfermagem da Faculdade Santo Agostinho (FSA), ${ }^{* *}$ Enfermeira, mestranda do Programa de Pósgraduação em Enfermagem (PPGENF), pela Universidade Federal do Piaui (UFPI), ***Biólogo, Docente da Faculdade Santo Agostinho, Especialista em Educação Ambiental, Doutorando em Biologia Celular e Molecular Aplicada à Saúde - ULBRA/RS

\section{Resumo}

Objetivo: Descrever e analisar a importância da atuação do enfermeiro na prevenção da gangrena de Fournier com ênfase na saúde do homem. Material e métodos: Trata-se de uma revisão integrativa de literatura, realizada no período de fevereiro a dezembro de 2013, através de consultas nas bases de dados Lilacs, Pubmed, Scielo, e Bdenf. Resultados: Foram encontrados 28 artigos, classificados em artigos médicos e de enfermagem. A análise das publicaçóes selecionadas permitiu a identificação de duas categorias temáticas: fatores que influenciam os homens a serem a população mais acometida pela gangrena de Fournier e a atuação do enfermeiro na prevenção dessa doença nos homens. Conclusão: É importante que o enfermeiro atue na prevenção da gangrena de Fournier promovendo educação em saúde e conhecimento de forma dinâmica do público masculino, de modo a minimizar a resistência por parte dos homens em procurar um serviço de saúde.

Palavras-chave: enfermeiro, gangrena de Fournier, saúde do homem.

\section{Abstract}

\section{Nurse acting in the prevention of Fournier's gangrene: attention to men's health}

Objective: To describe and analyze the importance of the work of nurses in the prevention of Fournier gangrene with emphasis on human health. Methods: It is an integrative literature review conducted during February to December 2013, through consultations in Lilacs, Scielo, and Bdenf data. Results: 28 items, sorted in medical and nursing articles were obtained. The analysis of selected publications allowed the identification of two thematic categories: factors that influence men to be the population most affected by Fournier's gangrene and the nurses in the prevention of Fournier gangrene in men. Conclusion: It is important for nurses to act in preventing Fournier gangrene with promotion in health education and promotes this knowledge in a dynamic way with the male audience in order to minimize resistance by men to seek a health service.

Key-words: nurse, Fournier gangrene, men's health. 


\section{Resumen}

\section{Actuación del enfermero en la prevención de la gangrena de Fournier: atención a la salud del hombre}

Objetivo: Describir y analizar la importancia de la actuación de los enfermeros en la prevención de la gangrena de Fournier con énfasis en la salud del hombre. Métodos: Se trata de una revisión integradora de la literatura realizada entre febrero y diciembre de 2013, a través de consultas en las bases de datos Lilacs, Scielo y Bdenf. Resultados: Fueron encontrados 28 estudios, clasificados en investigaciones médicas y de enfermería. El análisis de las publicaciones seleccionadas permitió la identificación de dos categorías temáticas: factores que influencian los hombres a ser la población más afectada por la gangrena de Fournier y la actuación del enfermero en la prevención de esa enfermedad. Conclusión: Es importante que el enfermero actúe en la prevención de gangrena de Fournier con la promoción de la educación para la salud y promover el conocimiento de una manera dinámica con el público masculino, con el fin de minimizar la resistencia de los hombres a buscar un servicio de salud.

Palabras-clave: enfermero, gangrena de Fournier, salud del hombre.

\section{Introdução}

A Gangrena de Fournier (GF) constitui-se de um grande problema de saúde pública, pois é uma doença pouca conhecida pelos profissionais da saúde, especificamente os enfermeiros e por apresentar significativas taxas de mortalidade masculina. Apesar disso, as açóes de prevençóes voltadas para essa doença vêm sendo pouco exploradas, favorecendo o surgimento de novos casos e o agravamento do quadro clínico. Por isso, é importante que o enfermeiro trabalhe com medidas preventivas e que também detecte precocemente a gangrena, auxiliando no enfrentamento da doença com a redução de suas complicaçóes.

Para conceituar a GF, autores afirmam tratar-se de uma infecção grave, podendo ser observada após procedimentos urológicos, proctológicos ou ginecológicos. Associa-se também a algumas doenças como diabetes mellitus (60\%). Além disso, idade avançada, hospitalização prolongada, carcinoma, alcoolismo, imunossupressão, dentre outros, também são mencionados [1].

É uma afecção de rápida progressão, com dados significativos de morbimortalidade, estimada em $20 \%$ [2]. O prognóstico satisfatório para esta gangrena também está baseado nos cuidados de enfermagem, por esta se encontrar mais próxima do paciente, neste sentido é de fundamental importância que a enfermagem tenha conhecimento claro sobre essa doença, bem como suas principais complicaçóes e, também, por se tratar de uma doença mutiladora, que acarreta problemas na autoestima, resultando em sentimento de medo e insegurança.

A GF já foi detectada em crianças e mulheres, mas sua prevalência é bem maior nos homens, isso se deve ao fato de os homens ainda acreditarem na assistência curativista e só procurarem o serviço de saúde quando a doença está instalada e em estágio avançado [3].

Para caracterizar esse comportamento dos homens, Alves et al. [4] explicam a resistência masculina aos serviços de saúde e apontam como razão as barreiras socioculturais e institucionais. Para o Ministério da Saúde, essa resistência está relacionada aos estereótipos de gêneros, por produzirem práticas baseadas em crenças e valores do que é ser masculino. Afirma também que o homem se julga ser invulnerável, contribuindo para que ele se cuide menos e consequentemente se expondo às situaçôes de risco [5].

Dentro desta perspectiva, o enfermeiro tem sua contribuição importante e relevante à medida que oferece eficiência e responsabilidade no desempenho de suas atividades, agindo de forma humanizada, técnica, competente, acolhedora, capaz de entender e escutar [6].

Diante desta abordagem o estudo tem como objetivo, descrever a produção científica acerca do papel do enfermeiro na prevenção da gangrena de Fournier relacionada à saúde do homem, e com isso, analisar aspectos importantes sobre o papel do enfermeiro na prevenção da GF com ênfase na saúde do homem.

\section{Material e métodos}

O presente estudo tratou-se de uma revisão integrativa de literatura, com abordagem qualitativa. O levantamento bibliográfico foi realizado no período de fevereiro a dezembro de 2013, por meio da consulta direta de livros específicos em bibliotecas e pela internet, no endereço eletrônico da 
plataforma da Biblioteca Virtual de Saúde (BVS), nas seguintes bases de dados: Lilacs, Scielo, Bdenf e Pubmed. Utilizaram-se como critério de inclusão os estudos que tinham entre seus descritores: saúde do homem, gangrena de Fournier e Enfermagem; resumos ou artigos em português, inglês e espanhol que abordassem a temática em estudo independente do método de pesquisa utilizado; descritos na íntegra e publicados pelo menos nos últimos 6 anos.

Como critério de exclusão, optou-se por não utilizar artigos que náo correspondiam ao objeto de estudo, textos que se encontravam incompletos, artigos que não estivessem disponíveis na íntegra online, que não forneciam informações suficientes para a temática e aqueles que não possuíam os descritores determinados pelos pesquisadores.

\section{Resultados e discussão}

Durante a elaboração da pesquisa fez-se um levantamento das publicaçóes relevantes ao tema e foram encontrados 14867 estudos. Após este levantamento, utilizou-se como critério de escolha os estudos publicados nos anos de 2008 a 2013, com prevalência em idiomas em português, seguidos de inglês e espanhol, textos completos e que fossem relevantes ao tema. Com isso obteve-se 28 publicaçóes refinados de acordo com os objetivos do estudo e distribuídos em diferentes periódicos.

A pesquisa foi realizada utilizando-se os descritores DECS/MESH: Enfermagem (Nursing), Gangrena de Fournier (Fournier's Gangrene/La Gangrena de Fournier), Saúde do Homem (Men's Health), realizando descritores individuais e cruzamentos duplos. A síntese dos descritores utilizados, das bases de dados e das referências selecionadas está descrita na tabela abaixo:

Tabela I - Distribuição das referências obtidas nas bases de dados Pubmed, Lilacs, Scielo, Bdenf, seguindo os descritores estabelecidos. Teresina, 2013.

\begin{tabular}{clcc}
\hline $\begin{array}{c}\text { Base de } \\
\text { dados }\end{array}$ & Descritores cruzados & $\begin{array}{c}\text { Referências } \\
\text { obtidas }\end{array}$ & $\begin{array}{c}\text { Refe- } \\
\text { rências } \\
\text { selecio- } \\
\text { nadas }\end{array}$ \\
\hline Scielo & $\begin{array}{l}\text { Enfermagem/Saúde } \\
\text { do Homem } \\
\text { Enfermagem/Saúde } \\
\text { Lilacs }\end{array}$ & 8192 & 1 \\
Bdenf & $\begin{array}{l}\text { Enfermagem/Saúde } \\
\text { do Homem }\end{array}$ & 1679 & 2
\end{tabular}

$\begin{array}{llcl}\text { Pubmed } & \text { Fournier's Gangrene } & 976 & 7 \\ \text { Scielo } & \text { Gangrena de Fournier } & 23 & 7 \\ \text { Lilacs } & \text { Gangrena de Fournier } & 31 & 3 \\ \text { Scielo } & \text { Enfermagem } & 37 & 4 \\ \text { Lilacs } & \text { Enfermagem } & 8921 & 2\end{array}$

Fonte: Biblioteca Virtual em Saúde - BVS

A maioria dos artigos selecionados foi publicada por médicos, totalizando 19 artigos; os demais eram publicaçóes de enfermeiros com 9 artigos. Com relação ao idioma, 17 artigos encontravam-se em português, 7 em inglês e 4 em espanhol. Os estudos ainda foram observados quanto ao tipo de estudos onde, a abordagem qualitativa foi a mais evidente em 20 artigos, 1 artigo com abordagem quantitativa e 7 artigos quanti-qualitativos. A listagem dos artigos encontra-se na tabela II.

Tabela II - Distribuição das referencias incluídas na revisão integrativa, de acordo com as bases de dados, ano, idioma, área de atuação e tipo de estudo. Teresina, 2013

\begin{tabular}{|c|c|c|c|}
\hline $\begin{array}{c}\text { Base de } \\
\text { dados }\end{array}$ & Idioma & Área & Tipo de estudo \\
\hline Lilacs & Port. & Enfermeiro & Quali \\
\hline Scielo & Port. & Médico & Quali-Quanti \\
\hline Pubmed & Ing. & Médico & Quali-Quanti \\
\hline Pubmed & lng. & Médico & Quali \\
\hline Lilacs & Port. & Enfermeiro & Quali \\
\hline Bdenf & Port. & Enfermeiro & Quali \\
\hline Lilacs & Port. & Médico & Quali \\
\hline Scielo & Port. & Médico & Quali \\
\hline Lilacs & Esp. & Médico & Quali \\
\hline Scielo & Esp. & Médico & Quali-Quanti \\
\hline Scielo & Port. & Médico & Quali-Quanti \\
\hline Lilacs & Port. & Médico & Quali \\
\hline Scielo & Esp. & Médico & Quali-Quanti \\
\hline Scielo & Port. & Enfermeiro & Quali \\
\hline Bdenf & Port. & Enfermeiro & Quali \\
\hline Scielo & Port. & Médico & Quali \\
\hline Scielo & Esp. & Médico & Quanti \\
\hline Pubmed & Ing. & Médico & Quali-Quanti \\
\hline Scielo & Port. & Enfermeiro & Quali \\
\hline Lilacs & Port. & Enfermeiro & Quali \\
\hline Scielo & Port. & Enfermeiro & Quali \\
\hline Pubmed & Ing. & Médico & Quali \\
\hline Lilacs & Port. & Médico & Quali-Quanti \\
\hline Pubmed & Ing. & Médico & Quali \\
\hline Pubmed & Ing. & Médico & Quali \\
\hline Pubmed & Ing. & Médico & Quali \\
\hline Scielo & Port. & Enfermeiro & Quali \\
\hline
\end{tabular}


De um modo em geral, os estudos selecionados retratam a atuação do enfermeiro na detecção precoce da GF, ações de prevenção na atenção primária, abordagem na educaçáo em saúde, quais os fatores de risco para o aparecimento da GF, o motivo da prevalência da doença em homens, as políticas de saúde voltadas ao público masculino, dentre outros.

De acordo com o levantamento realizado nas bases de dados, Scielo, Lilacs, Pubmed e Bdenf, contemplaram-se as produçóes mais atualizadas acerca da atuação do enfermeiro na prevenção da GF com ênfase na saúde do homem, conforme apresentado na Tabela III.

Tabela III - Distribuição das publicações conforme ano e titulo do periódico. Teresina, 2013.

\begin{tabular}{|c|c|c|c|}
\hline Ano & Periódico & $\begin{array}{l}\text { Frequên- } \\
\text { cia }\end{array}$ & $\%$ \\
\hline 2008 & $\begin{array}{l}\text { Revista da Rede de Enferma- } \\
\text { gem do Nordeste }\end{array}$ & 01 & 3,5 \\
\hline 2009 & Revista Enfermagem UERJ & 01 & 3,5 \\
\hline 2009 & $\begin{array}{l}\text { Revista Brasileira de Coloproc- } \\
\text { tologia }\end{array}$ & 01 & 3,5 \\
\hline 2009 & Canadian Family Physician & 01 & 3,5 \\
\hline 2009 & $\begin{array}{l}\text { Case in Reports Gastroente- } \\
\text { rology }\end{array}$ & 01 & 3,5 \\
\hline 2010 & Revista Enfermagem UERJ & 01 & 3,5 \\
\hline 2010 & $\begin{array}{l}\text { Interface - comunicação, saú- } \\
\text { de, educação }\end{array}$ & 01 & 3,5 \\
\hline 2010 & $\begin{array}{l}\text { Revista Brasileira de Coloproc- } \\
\text { tologia }\end{array}$ & 01 & 3,5 \\
\hline 2010 & $\begin{array}{l}\text { Cuadernos de Cirugía (Val- } \\
\text { divia) }\end{array}$ & 01 & 3,5 \\
\hline 2010 & $\begin{array}{l}\text { Revista do Colégio Brasileiro } \\
\text { de Cirurgiões. }\end{array}$ & 01 & 3,5 \\
\hline 2010 & Revista Chilena de Infectología & 01 & 3,5 \\
\hline 2010 & Revista da Imagem (online). & 01 & 3,5 \\
\hline 2010 & Revista Chilena de Cirugía. & 01 & 3,5 \\
\hline 2011 & $\begin{array}{l}\text { Revista Latinoamericano de } \\
\text { Enfermagem }\end{array}$ & 01 & 3,5 \\
\hline 2011 & $\begin{array}{l}\text { REME - Revista Mineira de } \\
\text { Enfermagem }\end{array}$ & 01 & 3,5 \\
\hline 2011 & Ciência \& Saúde Coletiva & 01 & 3,5 \\
\hline 2011 & Revista Chilena de Cirugía & 01 & 3,5 \\
\hline 2011 & $\begin{array}{l}\text { Revista Brasileira de Cirurgia } \\
\text { Plástica }\end{array}$ & 01 & 3,5 \\
\hline 2011 & $\begin{array}{l}\text { Case Reports in Emergency } \\
\text { Medicine }\end{array}$ & 01 & 3,5 \\
\hline 2012 & Ciência Saúde Coletiva & 01 & 3,5 \\
\hline 2012 & $\begin{array}{l}\text { Escola Anna Nery Revista de } \\
\text { Enfermagem }\end{array}$ & 01 & 3,5 \\
\hline
\end{tabular}

\begin{tabular}{llll}
2012 & $\begin{array}{l}\text { Revista da Escola de Enferma- } \\
\text { gem da USP }\end{array}$ & 01 & 3,5 \\
2012 & Case Reports in Urology & 01 & 3,5 \\
2012 & $\begin{array}{l}\text { Revista Brasileira de Cirurgia } \\
\text { Plástica }\end{array}$ & 01 & 3,5 \\
2013 & Case Reports in Pediatrics & 01 & 3,5 \\
2013 & Bmc Infectious Diseases & 01 & 3,5 \\
2013 & World Journal of Emergency & 01 & 3,5 \\
2013 & Surgery - WJES & 01 & 3,5 \\
\hline
\end{tabular}

Fonte: Banco de dados Biblioteca Virtual em Saúde.

Através da análise dos dados coletados, foi possível observar que em 2010 (28,6\%) houve uma apresentação maior de publicaçóes de interesse dos pesquisadores em publicar estudos que abordassem o foco da temática.

Quanto ao periódico, percebeu-se uma quantidade igual de publicaçóes nas revistas Ciência \& Saúde; Revista de Enfermagem UERJ, Revista Brasileira de Coloproctologia; Revista Chilena Cirugía; Revista Brasileira de Cirurgia Plástica, com representação de $7,14 \%$ cada.

Quanto à metodologia do estudo, observou-se uma diversidade nos estudos analisados, caracterizando a maior frequência de estudo de caso (54\%), e a pesquisa de campo, análise de prontuários e revisão integrativa compreendendo os outros $46 \%$.

Diante das apresentaçóes das publicaçóes o estudo permitiu fazer uma análise de duas categorias temáticas que estão descritas abaixo:

\section{Fatores que influenciam os homens a ser a popu- laçáo mais acometida pela gangrena de Fournier}

Nesta categoria foram selecionados 17 estudos. De acordo com alguns autores, a gangrena de Founier é uma infecção polimicrobiana, causada por bactérias aeróbias e anaeróbias que atuam de modo sinérgico, levando a uma fascite necrosante que acomete as regióes genital, perineal e perianal. Como consequência, tem-se uma endarterite obliterante que resulta na trombose dos vasos e necrose da regiáo afetada, é também considerada de evolução rápida e podendo levar a graves complicaçóes como atingir as regióes abdominal, retroperitônio e dorsal, falência múltipla de órgãos, sepse e até a morte [7-10].

Foi descrita em ambos os gêneros e em todas as faixas etárias, mas afeta predominantemente os homens. Isso se explica pelo fato dos homens apre- 
sentarem alguma patologia agregada, e pelas condições de saúde nas quais são submetidos $[3,11,12]$.

A GF torna-se mais agressiva e destrutiva quando há presença de uma lesão local associada a outras patologias que servem como fatores de risco da doença, dentre elas estão, diabetes mellitus, alcoolismo, neoplasias, HIV, imunossupressão, idade avançada, intervenção cirúrgica perirretal ou perineal, infecção geniturinária, desnutrição e falência renal. A sepse é a complicação mais frequente nos indivíduos que vão a óbito, e o índice de letalidade está na grande maioria relacionada à infecção sistêmica $[1,3,13]$.

A hemorroidectomia foi apresentada em alguns casos como fator para o surgimento da GF, porém ainda é considerada rara e inesperada devido à ausência de fatores predisponentes, e isso ocorre em decorrência de uma complicação pós-cirúrgica ou diagnóstico tardio [14].

A GF foi relatada também por Tsinti et al. [15] por apresentar maior predisposiçáa a pessoas com infecçôes virais, descrevendo as lesóes por HPV um forte fator para o seu desenvolvimento. Outro fator predisponente relatado na literatura por Khemees et al. [16], que considera a bactéria Neisseria meningitidis causadora da GF, ocasionando a uretrite que tem uma alta incidência em homens homossexuais devido a alteraçóes no comportamento sexual.

A GF é considerada uma doença de progressão rápida e agressiva de acordo com suas manifestaçóes clínicas, podendo influenciar na detecção do diagnóstico, e com consequência elevar o número de pessoas acometidas pela gangrena. $\mathrm{O}$ diagnóstico deve ser feito através do exame clínico, exames de imagem, e exames laboratoriais. Tendo em vista, uma falha neste diagnóstico, pode comprometer a sobrevida do paciente [17].

Além do tratamento da infecção com o uso dos antibióticos, é imprescindível tratar a ferida, pois estes não penetram em alguns tecidos, necessitando a realização da retirada dos tecidos desvitalizados. Dependendo da extensão da lesão, faz-se necessário a reconstrução desses tecidos, através da orquiectomia, enxerto de pele, amputação dos testículos e retalhos cutâneos e fasciocutâneo [17].

Outro tratamento que se mostrou bastante eficaz foi a oxigenoterapia hiperbárica, diminuindo sua mortalidade [7]. Em complemento, autores [2] relatam, em alguns casos, que o tratamento se baseia também na orquiectomia bilateral, penectomia total, transversosostomia em alça e a cistostomia.
É uma doença que apresenta altos índices de mortalidade, apesar de avanços terapêuticos existentes, e tem como medidas essenciais para se tentar diminuir esses altos índices de mau prognósticos, o reconhecimento precoce associado ao tratamento agressivo e invasivo, juntamente com o uso de antibióticos de amplo espectro [19,20].

\section{A atuaçáo do enfermeiro na prevençáo da gan- grena de Fournier nos homens}

Para a análise dessa categoria foram selecionados 11 artigos e 2 políticas que retratassem o trabalho do enfermeiro dentro da ESF e suas medidas para a prevenção e controle das doenças, especificamente no público masculino. Embora o estudo tenha como enfoque o trabalho preventivo na GF, não será possível descrever este trabalho especifico na Gangrena de Fournier devido ao fato de náo haver estudos publicados por enfermeiros fazendo este trabalho. Com isso, decidiu-se analisar o enfermeiro nas práticas preventivas as doenças, trabalhando a educação em saúde nos homens $\mathrm{e}$ aliando suas atividades preventivas com a gangrena.

De acordo, com a atuaçáo do enfermeiro dentro da equipe de saúde, é necessário que o enfermeiro para desenvolver suas atividades preventivas conheça a política. Com isso, a Política Nacional de Atenção Básica preconiza a saúde em seu âmbito individual e coletivo e em todos seus segmentos como a promoção e proteção da saúde, prevenção dos agravos, diagnóstico, tratamento, reabilitação e manutenção da saúde [21].

Diante desta política, o enfermeiro atuante na equipe de saúde deverá conhecer as reais necessidades da população de sua área de atuação, devendo o mesmo observar qual o maior motivo que leva as pessoas a procurarem o serviço de saúde, ter diálogo com a população, fazer um levantamento das condiçóes sociais e de dados epidemiológicos que interferem no processo saúde-doença daquela comunidade. Todos esses fatores contribuem para que o profissional possa conhecer e entender as necessidades da população e, com isso, traçar metas para minimizar esses problemas [6,22,23].

Dentro desta perspectiva, a principal ferramenta de trabalho que valorize o ser humano dentro deste contexto é a educação em saúde, que para a realização de açóes educativas em saúde praticadas pelos enfermeiros dentro da comunidade tem em seus objetivos a participação da população nas 
açóes de saúde, na compreensão das informaçóes, tornando-os seres capazes de identificar os problemas de saúde e principalmente cuidar da própria saúde $[24,25]$.

Um desafio a ser enfrentado pelo enfermeiro dentro da comunidade é fazer com que os homens compreendam a importância de manter padróes de qualidade de vida, procurar os serviços de saúde náo somente quando a doença estiver instalada. Este comportamento masculino de resistência à atenção básica se explica através da Politica Nacional de Atenção Integral à Saúde do Homem, na qual explicam o fato da dificuldade de acesso ao serviço de saúde e principalmente ao fator cultural de que o processo de adoecimento é considerado algo frágil e que o ser masculino se julga invulnerável $[5,26]$.

A partir daí cabe ressaltar a necessidade de o enfermeiro na atenção básica começar a despertar o interesse em conhecer as políticas e os programas voltados à população masculina e focar sua estratégia de prevenção, a fim de atender as necessidades dessa população [27].

A atuação do enfermeiro na prevenção da GF nos homens deve ser pautada no respeito e na confiança, de modo a criar um vínculo entre o profissional e paciente e, assim, fazer com que os homens tenham uma melhor adesão ao serviço de saúde. No entanto, conforme observado foram identificados algumas deficiências da relação entre o enfermeiro e os homens, devido à ausência de acolhimento ou um acolhimento carente de qualificação profissional para lidar com este público. Outra deficiência pautada foi o fato da UBS ser considerada um espaço feminilizado, tanto pela maior frequência de mulheres quanto pelo número de profissionais em sua maioria ser composta de mulheres [28,29].

Considerar essas questóes e outras que podem estar ligadas a não procura dos homens pelo serviço tem como consequência aproximar os homens da atenção primária. Para isso, o enfermeiro deve em sua prática de educaçáo em saúde utilizar de estratégias didáticas, fazendo com que a educação deixe de ser um depósito de informaçóes e de estar centrada apenas na doença. No entanto, o objetivo maior da educação em saúde é fazer com que os indivíduos tornem-se capazes de cuidar de sua própria saúde e viver com qualidade de vida [30].

Enfim, o enfermeiro deve ter consciência de sua importância enquanto educador em saúde, por ser reconhecido como o profissional capaz de garantir a qualidade de vida da populaçáo e por compreender de forma holística e integral, e pela capacidade de acolher e reconhecer as necessidades da população [31].

\section{Conclusão}

Os estudos das publicações analisadas mostraram que a gangrena de Fournier é uma doença pouco conhecida, mais considerada grave devido sua cronicidade e altas taxas de mortalidade. Ocorre principalmente em indivíduos do sexo masculino e está associada a co-morbidades que predispóe o seu desenvolvimento.

Ficou evidente, na análise dos estudos desta pesquisa, a importância do enfermeiro na prevençáo da GF, pois como foi descrito é o profissional que está inserido na atenção básica que têm capacitação adequada para promover atividades de educação em saúde, com intuito de orientá-los sobre o seu risco, os danos causados pela sua agressividade e conscientizá-los da importância de se detectar precocemente esta enfermidade.

Portanto, para se realizar medidas de prevenção faz-se necessário o conhecimento da doença, entretanto, não foi possível descrever essas medidas preventivas específicas a GF devido à escassez de publicações de enfermeiros no trabalho preventivo. A falta de conhecimento dos profissionais de saúde é um fator agravante para a detecção precoce de uma patologia táo complexa como a GF. Porém, diante dos estudos analisados, foi possível detectar a importância do papel do enfermeiro na prevenção da GF como também sua importância em trabalhar açóes preventivas voltadas à saúde dos homens.

Constatou-se que é importante que o enfermeiro pratique educação em saúde, entenda a importância de executá-la com responsabilidade e dinamicidade, e acolha o público masculino de modo a estabelecer um vínculo profissional e usuário, e com isso diminuir a resistência por parte dos homens em procurar um serviço de saúde.

\section{Referências}

1. Candelária PAP, Klug WA, Capelhuchnik P, Fang CB. Síndrome de Founier: Análise dos fatores de mortalidade. Rev Bras Cloproct 2009;29(2):197-202.

2. Batista RR, Filho PRR, Castro CAT, Fonseca MFM, Albuquerque IC, Formiga GJS. Síndrome de Fournier secundária a adenocarcinoma de próstata avançado: relato de caso. Rev Bras Colo-proctol 2010;30(2): 228-31. 
3. Hota PK. Fournier's Gangrene: Report of 2 Cases. Case Reports in Emergency Medicine. India, volume 2012, Article ID 984195, 4 pages, sep, 2011.

4. Alves RF, Silva RP, Ernesto MV, Lima AGB, Souza FM. Gênero e saúde: o cuidar do homem em debate. Psicol Teor Prat 2011;13(3):152-166.

5. Brasil. Ministério da Saúde. Política Nacional de Atenção Integral à Saúde do Homem: princípios e diretrizes. Brasília: MS; 2008.

6. Costa RKS, Miranda FAN. O enfermeiro e a estratégia saúde da família: contribuição para a mudança do modelo assistencial. Rev RENE 2008;9(2):120-28.

7. Rukshini P, Huey JCM, Pasupathy S. Necrotizing fasciitis. Canadian Family Physician 2009;55(10):981-7.

8. Garces SC, Gomez LMC, Florez GID, Munoz ZJD. Gangrena de Fournier en asociación con el uso de anti-inflamatorios no esteroideos. Reporte de un caso pediátrico. Rev Chil Infect 2010;27(4):341-44.

9. Dornelas MT, Correa MPD, Barra FML, Correa LD, Silva EC, Dornelas GV, et al. Síndrome de Fournier: 10 anos de avaliação. Rev Bras Cir Plást 2012;27(4):600-4.

10. Anantha RV, Kasper KJ, Patterson KG, Zeppa JJ, Delport J, Mccormick JK, Fournier's gangrene of the penis caused by Streptococcus dysgalactiae subspecies equisimilis: case report and incidence study in a tertiary-care hospital. BMC Infectious Diseases 2013;13(1):1-5.

11. Bocic AG, Abedrapo MM, Azolas MR, Llanos BJLB. Enfermedad de Fournier. Tratamiento. Rev Chilena de Cirugía 2010;62:635-8.

12. Cancino BC, Avendano HR, Poblete AC, Guerra HV. gangrena de Fournier. Cuad Cir (Valdivia) 2010;24(1):28-33.

13. Azolas MR. Factores de riesgo para mortalidad en gangrena de Fournier. Rev Chilena de Cirugía 2011;63(3):270-5.

14. Cakmak GK, Irkorucu O, Ucan BH, Karakaya K. Fournier's gangrene after open hemorrhoidectomy without a predisposing factor: Report of a case and review of the literature. Case Rep Gastroenterol 2009;3(2):147-55.

15. Tsinti M, Tsekoura T, Blevrakis E, Vlachakis I, Christaki AT. Fournier's gangrene associated with local cutaneous HPV lesions in a previously healthy girl. Case Reports in Pediatrics 2013.

16. Khemees TA; Porshinsky BS; Patel AP; Mcclung CD. Fournier's Gangrene in a Heterosexual Man: A Complication of Neisseria meningitidis Urethritis. Case Reports in Urology 2012.

17. Judice PLP, Christofoli MOJM, Oliveira PCR, Teles IG, Naijar YSJ. Gangrena de Fournier: relato de três casos, achados tomográficos e revisão da literatura. Rev Imagem (Online) 2010;32(1/2):21-23.
18. Mauro V. Retalho fasciocutâneo interna da coxa para reconstrução escrotal na síndrome de fournier. Revista Brasileira de Cirurgia Plástica 2011;26(4):707-9.

19. Mehl AA, Filho DCN, Mantovani LM, Grippa MM, Berger R, Krauss D, et al. Manejo da gangrena de Fournier: experiência de um hospital universitário de Curitiba. Revista do Colégio Brasileiro de Cirurgióes 2010;37(6):435-41.

20. Benjelloun EB, Souiki T, Yakla N, Ousadden A, Mazaz KM, Louchi A, et al. Fournier's gangrene: our experience with 50 patients and analysis of factors affecting mortality. World Journal of Emergency Surgery 2013;8(13).

21. Brasil. Ministério da Saúde. Política Nacional de Atenção Básica. Brasília: MS; 2012.

22. Schimith MD, Lima MADS. O enfermeiro na equipe de saúde da família: estudo de caso. Rev Enfermagem UERJ 2009;17(2):252-56.

23. Fortuna CM, Matumoto S, Pereira MJB, Mishina SM, Kawata LS, Borges CC. O enfermeiro e as práticas de cuidados coletivos na estratégia saúde da família. Rev Latinoam Enferm 2011;19(3):[8 telas].

24. Roecker S, Budó MLD, Marcon SS. Trabalho educativo do enfermeiro na estratégia saúde da família: dificuldades e perspectivas de mudanças. Rev Esc Enferm USP 2012;46(3):641-49.

25. Roecker S, Nunes EFPA, Marcon SS. Trabalho educativo do enfermeiro na estratégia saúde da família. Texto Contexto Enferm 2013;22(1):157-65.

26. Santana EM, Lima EMM, Bulhóes JLF, Monteiro EMLM, Aquino JM. A atenção à saúde do homem: açóes e perpectivas dos enfermeiros. Rev Min Enferm 2011;15(3):324-32.

27. Silva PAS, Furtado MS, Guilhon AB, Souza NVDO, David HMS. A saúde do homem na visão dos enfermeiros de uma unidade básica de saúde. Esc Anna Nery Enferm 2012;16(3):567-8.

28. Gomes R, Moreira MCNM, Nascimento EF, Rebello LEFS, Couto MT, Schraiber LB. Os homens não vêm! Ausência e/ou invisibilidade masculina na atenção primária. Ciênc Saúde Coletiva 2011;16(1):983-92.

29. Couto MT, Pinheiro TF, Valença O, Machin R, Silva GSN, Gomes R, et al. O homem na atenção primária à saúde: discutindo (in)visibilidade a partir da perspectiva de gênero. Interface - Comunic Saude Educ 2010;14(33):257-70.

30. Sousa LB, Torres CA, Pinheiro PNC, Pinheiro AKB. Práticas de educação em saúde no Brasil: a atuação da enfermagem. Rev Enferm UERJ 2010;18(1):55-60.

31. Backes DS, Backes MS, Erdmann AL, Buscher A. O papel do enfermeiro no sistema único de saúde: da saúde comunitária à estratégia de saúde da família. Ciênc Saúde Coletiva 2012;17(1):223-30. 